\title{
Proinflammatory gene expression in patients undergoing mitral valve surgery and maze ablation for atrial fibrillation
}

\author{
Feng-Chun Tsai, MD, ${ }^{\text {a }}$ Gwo-Jyh Chang, PhD, ${ }^{b}$ Yu-Juei Hsu, MD, PhD, ${ }^{c}$ Yuan-Min Lin, $\mathrm{PhD},{ }^{\mathrm{d}}$ \\ Yun-Shien Lee, PhD, ${ }^{e}$ Wei-Jan Chen, $\mathrm{MD}, \mathrm{PhD},{ }^{\mathrm{f}}$ Chi-Tai Kuo, $\mathrm{MD},{ }^{\mathrm{f}}$ and Yung-Hsin Yeh, $\mathrm{MD}^{\mathrm{f}}$
}

\begin{abstract}
Objective: It is difficult to achieve rhythm control in patients with long-standing persistent atrial fibrillation (AF). The radiofrequency maze procedure is an effective means in curing AF with a variable recurrence rate depending on patient characteristics and AF duration. In these patients, the characteristics of the atrial substrate have not been well investigated. Because the inflammatory process has been shown to be important in the pathogenesis of AF, we sought to characterize the proinflammatory gene expression in left atria obtained from patients with $\mathrm{AF}$ undergoing mitral valve surgery combined with the maze procedure to distinguish the changes associated with $\mathrm{AF}$ and its recurrence after the surgical ablation.
\end{abstract}

Methods: Left atrial appendages from 35 patients receiving mitral valve surgery were used for study. Ten patients had sinus rhythm (SR) and 25 patients had persistent $\mathrm{AF}$ for more than 1 year and underwent the maze procedure. Among the AF patients, 13 patients remained in SR (AF-SR) and 12 patients had recurrent $\mathrm{AF}$ during the 1-year clinical follow-up (AF-AF). The nCounter Human Inflammation Array (NanoString Technologies, Seattle, Wash) was used for evaluating proinflammatory gene expression. Quantitative polymerase chain reaction, Western blot, and immunohistochemistry were applied for studying messenger RNA and protein expression.

Results: Of 144 expressed proinflammatory genes, the inflammation array analysis revealed that 32 genes were differentially expressed between $\mathrm{AF}$ (including AF-SR and AF-AF) and SR. Thirteen genes were differentially expressed between AF-SR and AF-AF. The array and quantitative polymerase chain reaction produced parallel results in analyzing the expression of particular genes. Concordant with the gene expression difference between AF and SR patients, rapid pacing increased the expressions of $S H C 1, R H O A, P D G F A$, and TRAF2 in HL-1 myocytes, implicating a causative effect of tachyarrhythmia on these genes. Compared with AF-SR, AF-AF expressed more intense oxidative stress, upregulations of collagen, transforming growth factor beta 1 , and intranuclear nuclear factor of activated T-cells. Regression analysis showed that increased left atrial diameter was associated with the expression of RHOA and STAT1.

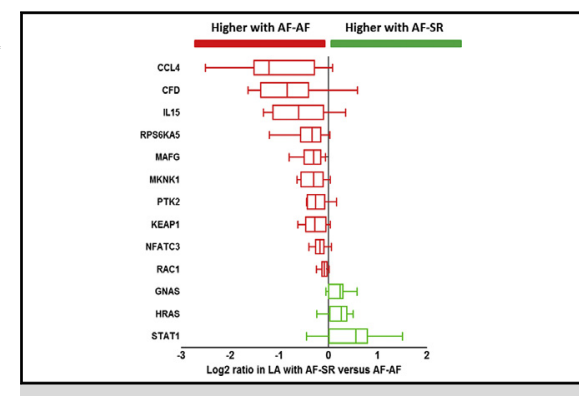

Gene expression in atrial fibrillation and its recurrence after surgical ablation.

Central Message

Proinflammatory gene expression in patients with AF undergoing maze ablation were characterized.

\section{Perspective}

Differential expression profiles of proflammatory genes were presented between sinus rhythm (SR) and atrial fibrillation (AF) and between maintained $\mathrm{SR}$ and recurrent $\mathrm{AF}$ after the maze procedure. The identified inflammatory molecules associated with $\mathrm{AF}$ and failed surgical ablation may provide clues for finding new potential therapeutic targets to improve $\mathrm{AF}$ rhythm control.

See Editorial Commentary page 1683.

\footnotetext{
From the ${ }^{\mathrm{a}}$ Division of Cardiac Surgery and ${ }^{\mathrm{f}}$ Cardiovascular Division, Chang-Gung Memorial Hospital, and ${ }^{\mathrm{b}}$ Graduate Institute of Clinical Medical Sciences, Chang-Gung University College of Medicine, Taoyuan, Taiwan; 'Division of Nephrology, Department of Medicine, Tri-Service General Hospital, National Defense Medical Center, Taipei, Taiwan; ${ }^{\mathrm{d} S c h o o l ~ o f ~ D e n t i s t r y, ~ N a t i o n a l ~}$ Yang-Ming University, Taipei, Taiwan; and ${ }^{\mathrm{e}}$ Department of Biotechnology, Ming-Chuan University, Taoyuan, Taiwan.

Supported by grants from the Chang-Gung Research Grant Foundation (Nos. CMRPG 3B0211-3, 3B0991-3, and 3C0621-2) and a Ministry of Science and Technology grant (No. 102-2314-B-182A-049).
}

Y-H. Yeh and C-T. Kuo contributed equally to this article.

Received for publication July 25, 2015; revisions received Nov 27, 2015; accepted for publication Dec 5, 2015; available ahead of print Jan 13, 2016.

Address for reprints: Yung-Hsin Yeh, MD, Cardiovascular Division, Chang-Gung Memorial Hospital, No. 5, Fu-Shin Rd, Kwei-Shan, Tao-Yuan, Taiwan 333 (E-mail: yeongshinn@cgmh.org.tw). $0022-5223 / \$ 36.00$

Copyright (c) 2016 by The American Association for Thoracic Surgery http://dx.doi.org/10.1016/j.jtcvs.2015.12.003 


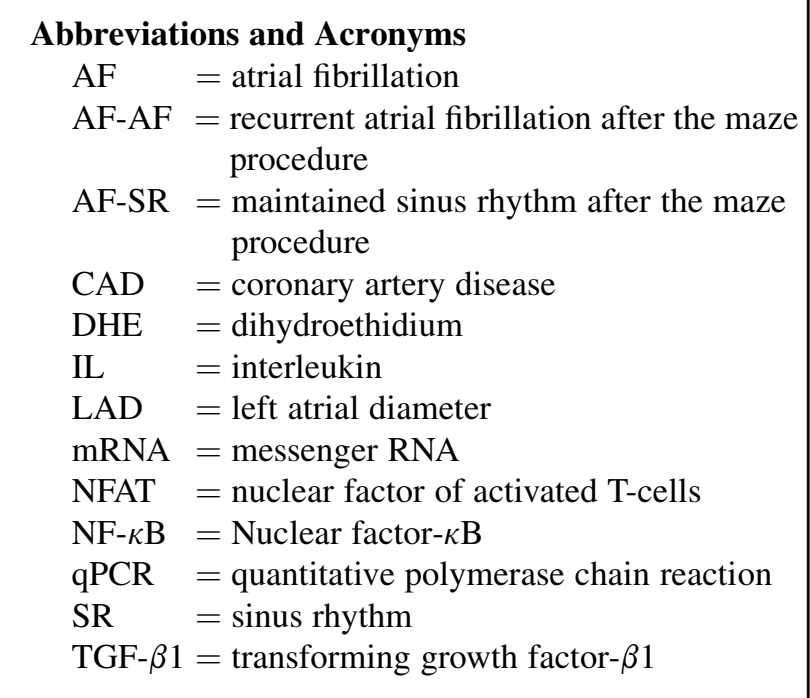

Scanning this QR code will take you to supplemental figures and tables for this article.

Conclusions: Differential expression profiles of proflammatory genes were presented between SR and AF and between maintained SR and recurrent AF after the maze procedure. The identified inflammatory molecules associated with $\mathrm{AF}$ and failed surgical ablation may provide clues for developing new potential therapeutic targets to improve AF rhythm control. (J Thorac Cardiovasc Surg 2016;151:1673-82)

Atrial fibrillation (AF) is the most common sustained arrhythmia. It is associated with increased risks of worsening heart failure, ischemic stroke, and death. As AF persists over time, atria substrate remodeling becomes more severe and rhythm control becomes more difficult (AF begets AF). ${ }^{1-3}$ Although major progress in $\mathrm{AF}$ rhythm control has been made mainly by catheter ablation during the past 20 years, $\mathrm{AF}$ recurrence is still a substantial clinical issue. In patients with longstanding $(>1$ year) persistent $\mathrm{AF}, \mathrm{AF}$ recurrence rate has been reported to be more than $50 \% 1$ year after single catheter ablation, which is quite disappointing. Clinical and animal studies have suggested that prolonged AF substantially results in more severe and frequently irreversible atrial remodeling regarding both gene expression changes and histologic features. ${ }^{4-6}$
Among patients undergoing mitral valve surgery, $\mathrm{AF}$ is associated with reduced survival and increased risk of stroke. Surgical ablation of AF is an effective treatment of rhythm control. The Cox-maze III surgical operation is the gold standard for surgical treatment of AF with a reported $\mathrm{AF}$ cure rate of $75 \%$ to $90 \%,{ }^{7,8}$ in contrast to the modified maze procedure, with a reported AF cure rate ranging from $44 \%$ to $90 \% .^{9-14}$ Several clinical factors have been demonstrated to be associated with $\mathrm{AF}$ recurrence after the maze procedure, including the preoperative duration of $\mathrm{AF}$, atrial size by echocardiography, and AF wave characteristics obtained during the cardiac surgery. ${ }^{15}$ However, the atrial substrate in these patients has not been well investigated. The maze procedure is highly effective in treating $\mathrm{AF}$, but the recurrence rate is still high in patients with markedly dilated atria. Enlarged atria are thought to be associated with more severe atrial remodeling, which increases the difficulties in treating AF. It could be helpful to improve the treatment if we find any molecular targets that can help distinguish failed and successful maze surgery in these patients with markedly dilated atria. Inflammation is a response that involves immune cells, blood vessels, and molecular mediators. The inflammatory process is widely involved in many human diseases, including cardiovascular disease. Numerous studies have shown mediators of the inflammatory response alter atrial electrophysiology and worsen structural substrates, thereby leading to increased AF vulnerability and refractoriness. ${ }^{16-18}$ This evidence includes elevated serum levels of inflammatory biomarkers, including C-reactive protein, tumor necrosis marker, interleukin (IL)-2, IL-6, and monocyte chemoattractant protein-1 in AF patients and upregulation of inflammatory mediators in the atria of $\mathrm{AF}$ patients and animal models of AF. Inflammation links to various pathologic processes, such as oxidative stress; electrical and calcium handling abnormalities; and fibrosis, which promotes atrial substrate remodeling. An improved understanding of the underlying pathophysiology should help improve the management options for AF. The inflammatory process is broadly diverse and contains numerous signaling pathways and mediators. In the past the inflammation-related studies in AF were mostly selective and have targeted genes or proteins of interest. Here we intended to find any potential targets and therefore chose a commercial set containing extensive inflammationrelated targets to screen a panel of genes that are likely involved in atrial remodeling. Therefore, in this study, we sought to characterize gene expression profiles of mediators and transcription factors relevant to inflammation in the atria among patients with preoperative sinus rhythm (SR), maintained SR, and recurrent AF at 1 year after the maze procedure in patients with mitral valve disease undergoing 
valvular surgery. Our data reveal that several transcriptional factors and mediators are differentially expressed among the 3 groups.

\section{MATERIALS AND METHODS Maze Ablation and Human Samples}

From September 2008 to August 2013, 261 patients with severe mitral valve disease and persistent $\mathrm{AF}$ with a duration known to be longer than 12 months underwent mitral valve surgery and maze procedure. Patients were operated on by a single surgeon with technical unity and the first 10 cases were excluded to avoid learning curve bias. Our routine approach was standard full sternotomy with bicaval cannulations, and $\mathrm{AF}$ ablation lesion sets were similar to traditional Cox-maze III, except most cut-and-sew lesions were replaced by the irrigated bipolar radiofrequency ablation device (Medtronic, Minneapolis, Minn) to create long continuous transmural lesions. Separated pulmonary vein isolation with 2 connecting lesions of both superior and inferior pulmonary veins were applied twice in both sides to confirm the complete ablation. Additional cryoablation of the mitral isthmus was applied with a cryoprobe $\left(-70^{\circ} \mathrm{C}\right.$ for 2 minutes). Biatrial ablation was applied in most cases and left atrial ablation alone was applied solely in elderly patients with isolated aortic valve replacement. The left atrial appendage was closed from inside with 4-0 Prolene (Ethicon, Inc, Cincinnati, Ohio) double running sutures and resected from outside for specimen analysis. The ligament of Marshall was divided during left pulmonary vein isolation and bilateral epicardial fats over the interatrial groove, composed of ganglional plexi, were also resected. Left atrial volume reduction for markedly enlarged left atrium, dimensions $>60 \mathrm{~mm}$ by preoperative echocardiography, was carried out with applications of posterior wall between the inferior pulmonary vein and mitral isthmus. Of these patients, sustained sinus rhythm, including sinus rhythm or an atrial-based paced rhythm, was present in $78 \%$ at 1 year. Between January 2011 and August 2013, 42 patients who agreed to participate in the study were enrolled and atrial specimens were obtained from these patients. These patients had no evidence of atrioventricular node dysfunction before surgery. Among these patients, 12 had recurrent $\mathrm{AF}$ and the others maintained sinus rhythm during 1-year follow-up. The mean \pm standard deviation left atrial diameter (LAD) by echocardiography was $58 \pm 10.1 \mathrm{~mm}$ for patients with recurrent $\mathrm{AF}$ and $48.3 \pm 13.0 \mathrm{~mm}$ for patients with maintained SR, respectively $(P=.065)$. AF recurrence rate is high in patients with markedly dilated atria. In this study we compared patients with more dilated atria with maintained SR $(n=13)$ and all enrolled patients with recurrent AF. Another 10 samples of left atrial appendage were obtained from patients with mitral valve disease and SR during the valvular surgery (SR group). Antiarrhythmic drugs were used by 6 patients of the maintained SR after the maze procedure group (AF-SR) who were free of AF. During follow-up at 1 year after the valvular surgery, cardiac echography showed no significant recurrent mitral regurgitation or stenosis in either the recurrent $\mathrm{AF}$ after the maze procedure (AF-AF) or AF-SR groups. The research protocol conformed to the ethical guidelines of the 1975 Declaration of Helsinki and was approved by the Chang-Gung Medical Foundation Institutional Review Board. Written informed consent was obtained from all participating patients.

\section{Proinflammatory Gene Expression Analysis}

Total RNA was isolated from left atrial appendages with the TRIzol reagent (Invitrogen, Carlsbad, Calif) and was further purified and concentrated by using the WelPrep tissue RNA kit (Welgene, Taipei, Taiwan). The quality and quantity of total RNA were analyzed by using a Bioanalyzer 2100 (Agilent Technologies, Santa Clara, Calif). Gene expression profiles in tissue RNA were analyzed with an nCounter Human Inflammation kit (NanoString Technologies, Seattle, Wash) following the manufacturer's protocols.

\section{Quantitative Polymerase Chain Reaction}

Total RNA was extracted by using the TRIzol reagent, and quantitative polymerase chain reaction (qPCR) was performed. The oligonucleotide sequences used are given in Table E1. Glyceraldehyde 3-phosphate dehydrogenase messenger RNA (mRNA) was used as the internal control.

\section{Confocal Immunohistochemistry}

Immunohistochemistry and quantitative assessment were carried out as described previously. ${ }^{19}$ Immunohistochemical analyses were performed using confocal microscopy (Leica TCSSP2, Mannheim, Germany) with collagen-1, transforming growth factor- $\beta 1$ (TGF- $\beta 1$ ), nuclear factor of activated T-cells cytoplasmic 3 (NFATc3), and vimentin (Sigma-Aldrich, St Louis, Mo) as primary antibodies, followed by fluorescein isothiocyanate (green) or Cy3 (red; Chemicon, Temecula, Calif) conjugated secondary antibodies. Nuclei were visualized by 40,6-diamidino-2-phenylindole staining. The expression of target proteins was calculated as protein-occupied area in the tissue divided by the nucleus area. For NFATc3, only the area within the nuclei was estimated. For each analysis, at least 5 random fields were chosen with $>30$ observations in each. Oxidative stress from reactive oxidative species in the tissues was measured with the fluorescent dye (dihydroethidium [DHE]) and was detected by using confocal microscopy. Samples were preincubated with or without $10 \mathrm{mmol} / \mathrm{L}$ DHE for 30 minutes. DHE was excited at $543 \mathrm{~nm}$ with an argon laser, and emission at $590 \mathrm{~nm}$ was recorded. Two-dimensional images $(512 \times 512$ pixels $)$ were acquired and analyzed with MetaMorph software (Universal Imaging Corp, West Chester, Pa).

\section{Western Blotting}

Proteins were extracted and processed as described previously. ${ }^{20}$ Briefly, specimens of the left atrial appendage were lysed with lysis buffer. After proteins were separated using sodium dodecylsulfate-polyacrylamide gel electrophoresis and transferred to polyvinylidene fluoride membranes, membranes were incubated overnight at $4^{\circ} \mathrm{C}$ with primary antibodies against RHOA, PDGFA, phospho-STAT1 (Millipore, Temecula, Calif), SHC1, TRAF2, CCL4, IL15, total STAT1, NFATC3 (Abcam, Cambridge, Mass), GAPDH, or tubulin (Santa Cruz, Dallas, Tex). Signals were detected by using electrochemiluminescence and were quantified using densitometry. Immunoreactive signal bands were in the linear range and expressed relative to GAPDH or tubulin. The software Image Gauge (Fuji Film, Tokyo, Japan) was used for the quantitative evaluation of the protein expression.

\section{Cell Culture and Tachypacing}

HL-1 atrial myocytes were maintained in Claycomb medium and subjected to field stimulation as described previously. ${ }^{19}$ HL-1 cells $(\geq 1 \times 106$ cells) on 4-well rectangular dishes (Nunclon, Rochester, NY) were placed into C-Dish 100 TM-Culture Dishes (IonOptix, Milton, Mass). HL-1 cells were then subjected to field stimulation with 10-ms stimuli of $40-\mathrm{V}$ intensity at 1,2 , and $4 \mathrm{~Hz}$ frequency for 24 hours $(1.5-\mathrm{V} / \mathrm{cm}$ field strength; C-Pace EP culture pacer, IonOptix). The capture efficiency $>90 \%$ was confirmed by microscopic examination.

\section{Statistical Analysis}

For patient characteristics, mean \pm standard deviation for continuous variables and count and percent for categorical variables are presented, respectively. Fisher exact test was used for category comparisons between groups. The data in the figures are presented with box-and-whisker plots. Unpaired Student $t$ test and 1-way analysis of variance with post hoc Tukey tests were applied for the 2 groups and multiple comparisons, respectively. For proinflammatory gene expression analysis, the data were normalized to the expression of housekeeping genes like GAPDH for each set and the significant difference between 2 groups was considered with a false 
TABLE 1. Demographic and clinical features of patients with sinus rhythm (SR), maintained SR (AF-SR) and recurrent atrial fibrillation (AF-AF) after undergoing a maze procedure

\begin{tabular}{|c|c|c|c|}
\hline Feature & $\begin{array}{c}\text { SR } \\
(\mathbf{n}=\mathbf{1 0})\end{array}$ & $\begin{array}{c}\text { AF-SR } \\
(n=13)\end{array}$ & $\begin{array}{c}\text { AF-AF } \\
(n=12)\end{array}$ \\
\hline \multicolumn{4}{|l|}{ Demographic } \\
\hline Age $(y)$ & $62.5 \pm 14.6$ & $56.6 \pm 11.6$ & $58 \pm 10.1$ \\
\hline Sex (M:F) & $5: 5$ & $5: 8$ & $8: 4$ \\
\hline \multicolumn{4}{|l|}{ Laboratory } \\
\hline $\begin{array}{l}\text { White blood cell count } \\
(1000 / \mu \mathrm{L})\end{array}$ & $6.6 \pm 1.8$ & $7.1 \pm 1.9$ & $6.9 \pm 2.1$ \\
\hline \multicolumn{4}{|l|}{ Clinical } \\
\hline Ejection fraction $(\%)$ & $48.4 \pm 24.3$ & $57.15 \pm 14.9$ & $65.4 \pm 10.3$ \\
\hline Left arterial diameter $(\mathrm{mm})$ & $46.3 \pm 9$ & $58.7 \pm 13.8^{*}$ & $56 \pm 10.9$ \\
\hline Rheumatic heart disease & 0 & $6^{*}$ & $5^{*}$ \\
\hline Hypertension & 6 & 4 & 2 \\
\hline Diabetes mellitus & 5 & 4 & 3 \\
\hline Coronary artery disease & 6 & $2^{*}$ & $1^{*}$ \\
\hline \multicolumn{4}{|l|}{ Medication } \\
\hline Aspirin & 6 & $2 *$ & $1^{*}$ \\
\hline
\end{tabular}

discovery rate $<5 \%$. Pearson correlation was used for correlation between expressions of proinflammatory gene and left atrial diameter.

\section{RESULTS}

Patient Characteristics and Proinflammatory Gene Expression Analysis

The clinical characteristics of patients are listed in Table 1. The age and left ventricular ejection fraction were not significantly different among SR, AF-SR, and AF-AF groups. The LAD as measured by 2-dimensional echocardiography was significantly smaller in SR patients compared with AF patients (including AF-SR and AF-AF). The LAD was not significantly different between AF-SR and AF-AF groups. There was no difference in sex between SR and AF patients, but there were more women enrolled in the AF-SR group compared with the AF-AF group. In patients with AF, 6 of 13 in AF-SR and 5 of 12 in AF-AF had rheumatic heart disease presenting with significant mitral stenosis ( $P$ value not significant). In $\mathrm{SR}$, more patients had coronary artery disease (CAD), hypertension, and diabetes mellitus compared with AF-SR and $\mathrm{AF}-\mathrm{AF}$.

Of the 184-probe sets, 144 genes were expressed at detectable levels (Table E2) in these samples. Among these, 32 genes were differentially expressed between SR and AF (including group AF-AF and AF-SR) (Figure 1). Fourteen genes were significantly upregulated in AF compared with SR, including HSPBI, STAT1, and PDGFA. Eighteen genes were significantly downregulated in $\mathrm{AF}$, including $I L 1 B$ and CXCL6. Thirteen genes were significantly differently expressed between group AF-AF and AF-SR (Figure 1). Ten genes were upregulated in AF-AF compared with AF-SR, including CCLA and IL15, whereas 3 of them were downregulated in AF-AF such as STATI.

In AF-AF group, 7 patients had recurrence of AF within the first month after the maze procedure. Recurrent $\mathrm{AF}$ was noted for the other 3,1 patient at 3,6, and 7 months, respectively. There was no association between the
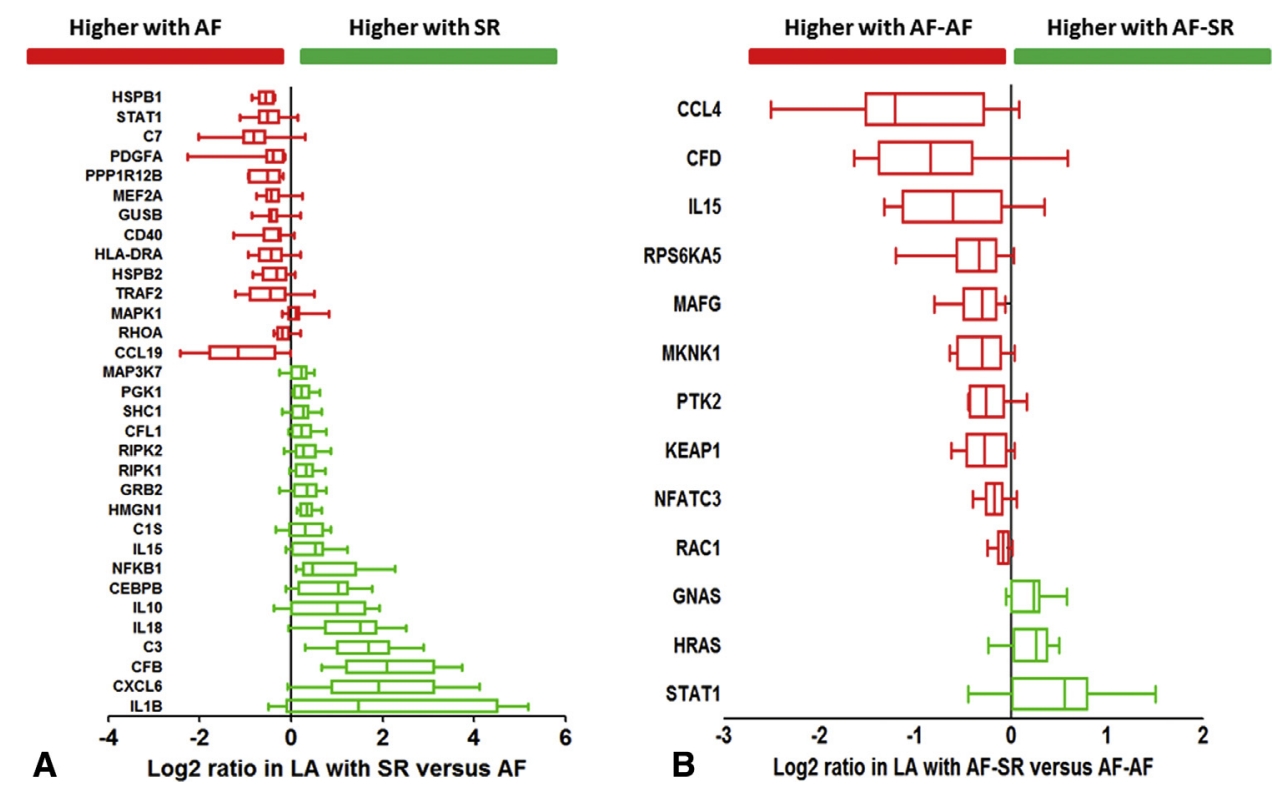

FIGURE 1. Significant differentially expressed proinflammatory genes in left atrial appendages (A) between sinus rhythm $(S R)$ and atrial fibrillation $(A F)$ and $(\mathrm{B})$ between recurrent $\mathrm{AF}$ after the maze procedure $(A F-A F)$ and maintained SR after the maze procedure $(A F-S R)$. The results are presented as log 2 (SR/AF) and $\log 2$ (AF-SR/AF-AF), respectively. $L A$, Left atria. 

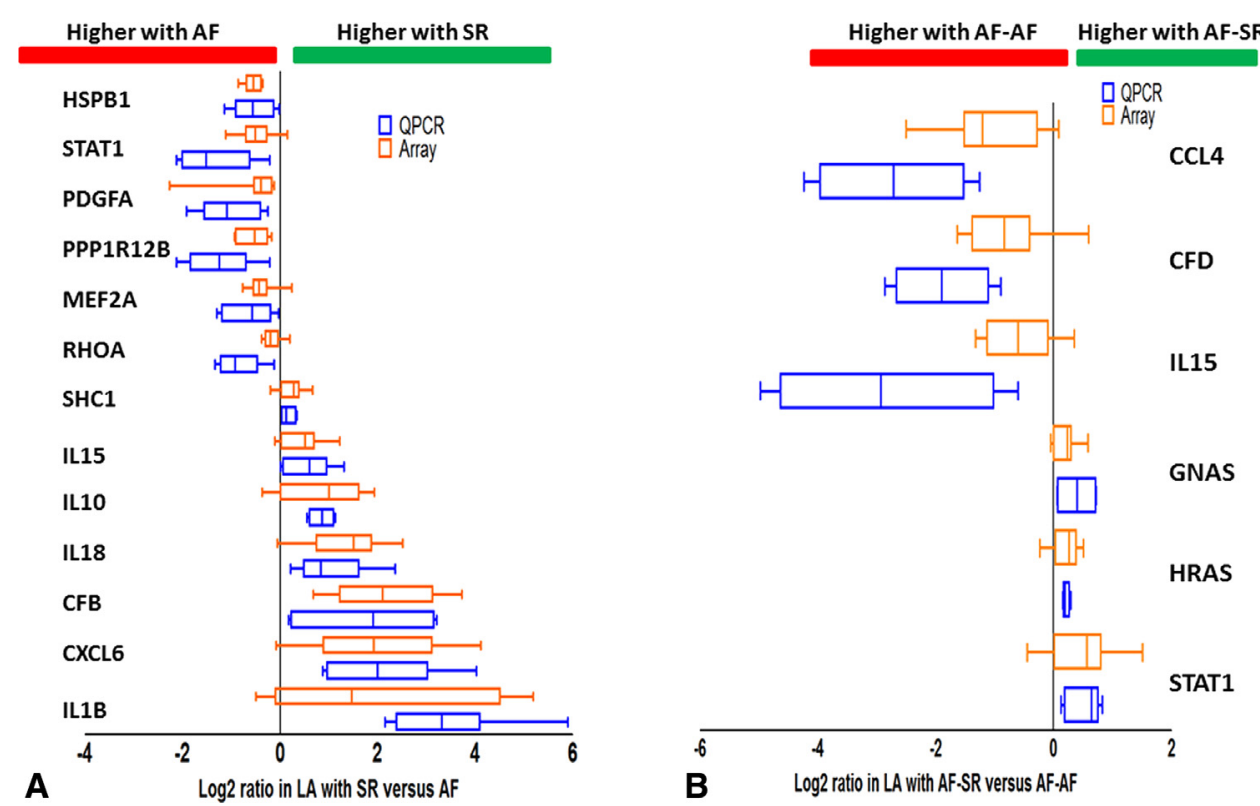

FIGURE 2. Comparison between quantitative polymerase chain reaction $(q P C R)$ and nCounter Human Inflammation Array (NanoString Technologies, Seattle, Wash) (A) between atrial fibrillation $(A F)$ and sinus rhythm $(S R)$ and (B) between recurrent AF after the maze procedure $(A F-A F)$ and maintained SR after the maze procedure $(A F-S R)$. Data are expressed as $\log 2$ fold change. Genes were selected by virtue of significant differences between groups in inflammation array analysis. The results were presented as $\log 2$ (SR/AF) and $\log 2$ (AF-SR/AF-AF), respectively. For qPCR, data are presented as box-andwhisker plot. $\mathrm{N}=5$ to 7 samples for each gene. $L A$, Left atria.

expression level of proinflammatory genes and AF-free duration in the group.

\section{qPCR}

To validate the inflammation array analysis, genes of interests were selected for qPCR analysis, which showed that the array and qPCR analyses produced parallel results with respect to the up- and downregulation of particular genes, but with modest differences in the magnitude of specific changes (Figure 2, $A$ and $B$ ).

\section{Protein Expression}

To evaluate whether the gene expression difference at transcriptional level was reflected on translational level, the expression of several genes were analyzed with Western blot. Consistent with the findings at the mRNA levels, we found that the expressions of SHC1, RHOA, PDGFA, and $T R A F 2$ were significantly increased in AF compared with SR (Figure 3, $A$, and Figure E1, $A$ ). Figure 3, $B$, and Figure E1, B, show the expressions of CCLA and IL15 were significantly increased in AF-AF compared with AF-SR. The expression of phopho-STATl was significantly reduced in AF-AF compared with AF-SR, whereas the total form of STATI was not different between the 2 groups. Although at the mRNA level both nuclear factor of activated T-cells, cytoplasmic (NFAT) and RAC1-related C3 botulinum toxin substrate 1 were differentially expressed between
$\mathrm{AF}-\mathrm{AF}$ and AF-SR, the expressions of both at the protein level were not significantly different.

\section{Ultrastructural Remodeling}

To evaluate if cardiac rhythm (AF vs SR) and $\mathrm{AF}$ refractoriness to the maze procedure are associated with the severity of atrial substrate remodeling, the expression of collagen I, TGF- $\beta 1$, NFATc 3 , and oxidative stress was evaluated by immunofluorescence. Figure 4 and Figure E2 showed that the expression of collagen, TGF- $\beta 1$, and oxidative stress was significantly increased in both AF-AF and AF-SR compared with SR. Moreover, the expressions of TGF- $\beta 1$ and oxidative stress were significantly higher in the atria of AF-AF compared with AF-SR, which implies that $\mathrm{AF}$ recurrence after surgical ablation is associated with more severe atrial substrate remodeling. NFAT is a nuclear transcription factor that has been shown to be involved in AF-related electrical and structural remodeling. Dephosphorylated NFAT was translocated into nucleus and module downstream gene expression. $^{21}$ Consistent with previous findings, we observed that the expression of intranuclear NFATc3 was more abundant in both AF-AF and AF-SR compared with $\mathrm{SR}$; furthermore, its expression was also significantly higher in AF-AF compared with AF-SR, suggesting that NFAT activation is associated with more severe $\mathrm{AF}$ remodeling and that it may contribute to AF refractoriness in the surgical ablation. 

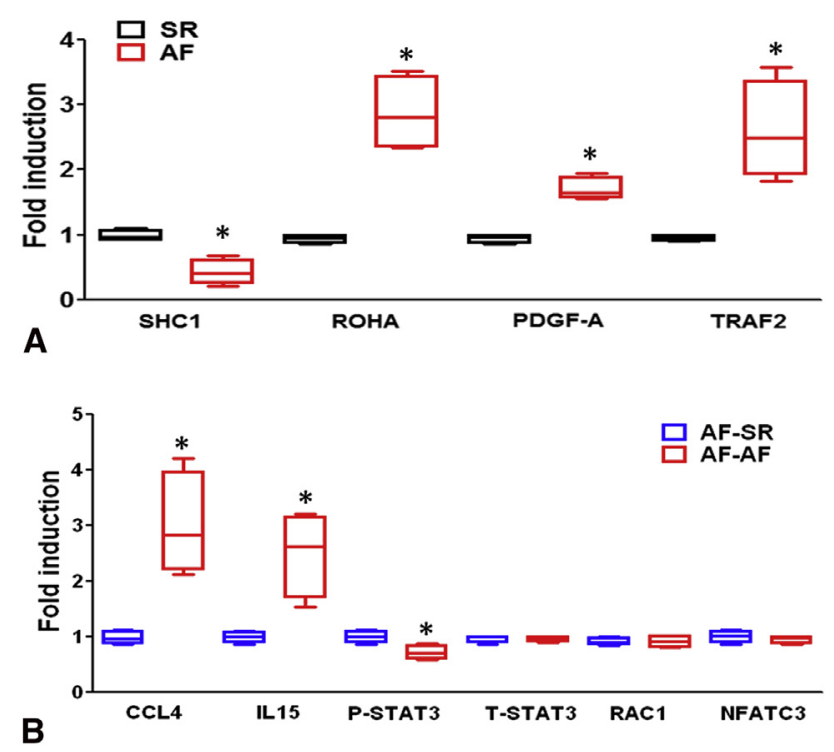

FIGURE 3. A, Western blot analysis of $S H C 1$, RHOA, PDGFA, and $T R A F 2$ between sinus rhythm $(S R)$ and atrial fibrillation $(A F)$. B, Western blot analysis of $C C L 4, I L 15$, phospho- and total STAT1, RAC1, and NFATc3 between recurrent $\mathrm{AF}$ after the maze procedure $(A F-A F)$ and maintained SR after the maze procedure $(A F-S R)$. The relative expression levels of proteins corresponding to $G A P D H$ were quantified by densitometry and normalized to A, SR and B, AF-SR, respectively, which was set at 1.0. $\mathrm{N}=3$ to 5 for each group. $* P<.05$.

\section{Tachypacing-Induced Gene Expression}

To determine if tachycardia have a causal relationship with the expression of the AF-associated remodeled genes, HL-1 myocytes were subjected to external electric activation at 1, 2, and $4 \mathrm{~Hz}$ for 24 hours. Figure 5 and Figure E3 indicated that the expression of PDGFA, $R H O A$, and TRAF2 at protein level was significantly increased, whereas $S H C l$ was reduced, by electric pacing in a frequency-dependent manner. The changes were

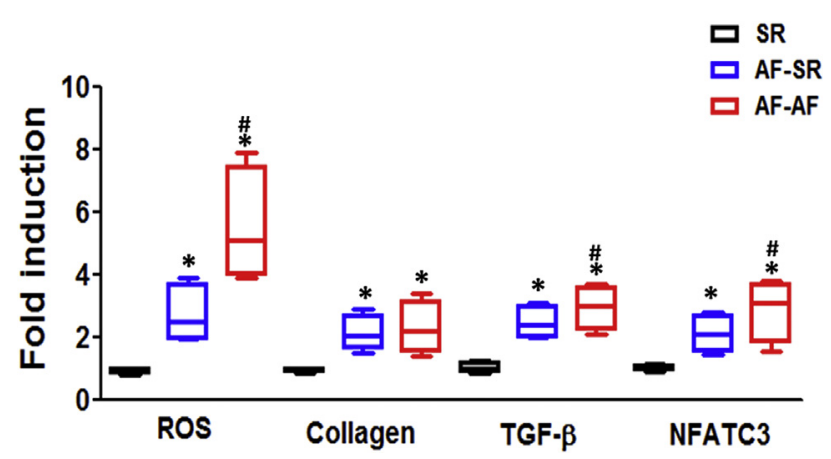

FIGURE 4. Confocal immunohistochemistry analysis of reactive oxidative stress $(R O S)$, transforming growth factor beta 1 (TGF- $\beta 1)$, collagen, and nuclear factor of activated T-cells cytoplasmic 3 (NFATC3) between sinus rhythm $(S R)$, maintained sinus rhythm after maze procedure $(A F-S R)$ and recurrent atrial fibrillation after maze procedure $(A F-A F)$. The relative intensity is normalized to $\mathrm{SR}$, which is set at $1.0 . \mathrm{N}=3$ to 5 samples for each group. *Versus SR. \#Versus AF-SR $(P<.05)$.

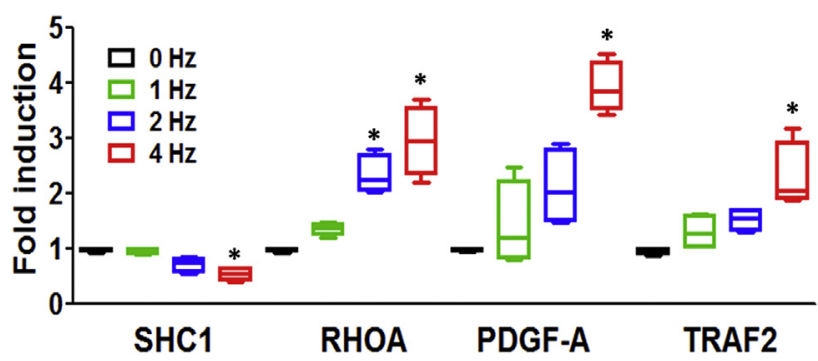

FIGURE 5. Activation of HL-1 atrial myocyte regulated the expression of $S H C 1, R H O A, P D G F-A$, and TRAF2 at protein level at a frequencydependent manner. The relative expression levels of proteins corresponding to $G A P D H$ were quantified by densitometry and normalized to $1-\mathrm{Hz}$ pacing, which was set at $1.0 . \mathrm{N}=4$ for each group. $* P<.05$ versus $1 \mathrm{~Hz}$.

similar to the findings in human AF versus SR, which implies that AF may have a causal relationship with the expression of these genes.

\section{Correlation Between Proinflammatory Genes and LAD}

The severity of atrial remodeling may be reflected in the degree of atrial dilatation. Because previous studies have shown that the atrial size is an independent factor predicting $\mathrm{AF}$ recurrence after the maze procedure, ${ }^{22,23}$ we evaluated the relationship between the expression of proinflammatory genes and the LAD by 2-dimensional echocardiography based on these specimens. Figure 6 shows that the expression of RHOA and STATI significantly correlated with the LAD. Consistent with prior research, our findings suggest that these proinflammatory molecules were likely involved in adverse remodeling associated with atrial dilation.

\section{Correlation Between Proinflammatory Genes and AF Recurrence After the Maze Procedure}

To evaluate if the expressions of proinflammatory genes help distinguish AF-AF and AF-SR in patients with markedly dilated atria, we performed the receiver operator curve analysis. The highest area under the curve is 0.82 for $P T K 2$ as shown in Figure 7 . The area under the curve is 0.763 for all of NFATC3, RAC1, and CCLA and 0.79 for STAT1, respectively.

\section{DISCUSSION \\ Main Findings}

Through an unadjusted screening of inflammationrelated genes, we found a differential gene expression profile in the left atria after the surgical ablation of SR versus $\mathrm{AF}$ and maintained $\mathrm{SR}$ versus $\mathrm{AF}$ recurrence. We further compared the characteristics of the atria between maintained SR versus AF recurrence after the surgical ablation and demonstrated that more intense structural remodeling in the atria of AF-AF compared with AF-SR, 

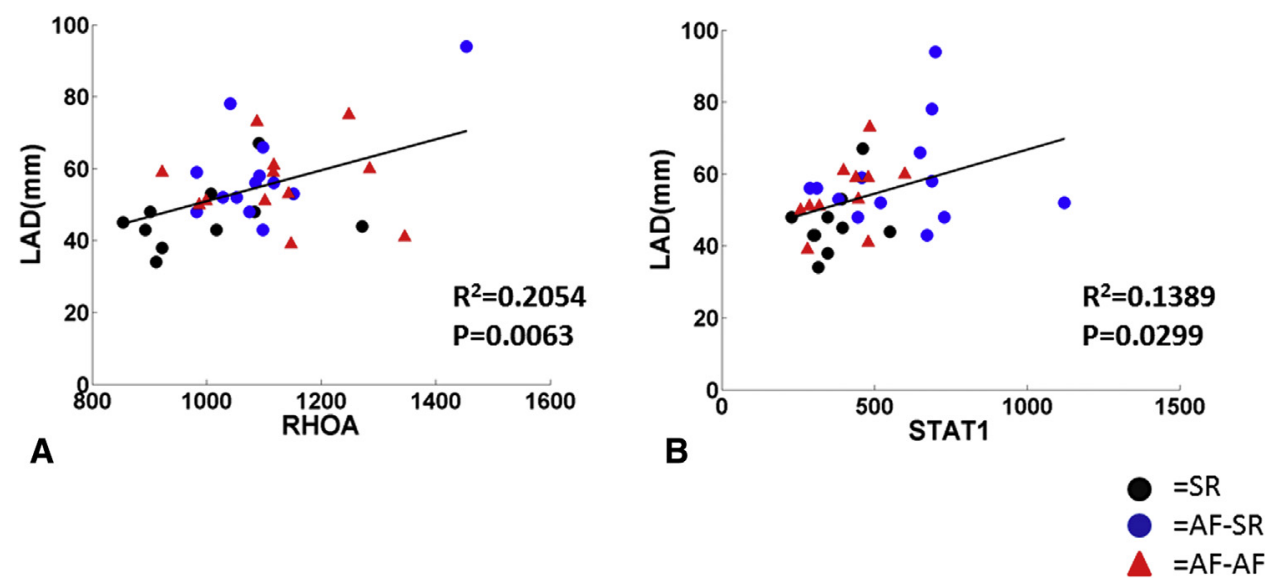

FIGURE 6. Regression analysis between left atrial diameter ( $L A D)$ and (A) RHOA and (B) STAT1. SR, Sinus rhythm; $A F-S R$, maintained sinus rhythm after maze procedure; $A F-A F, \mathrm{AF}$ recurrence after maze procedure.

further implying that severe structural remodeling as well as gene expression changes may underlie the failure to maintain sinus rhythm after surgical ablation. The results indicate that several inflammation-related transcription factors and mediators may present as potential targets in attenuating atrial remodeling by AF and improving AF therapy.

Like catheter ablation or use of antiarrhythmic drugs in treating $\mathrm{AF}$, the AF-free rate after the maze surgery is significantly reduced by the enlarged atria. It is generally agreed that a more dilated atria is associated with more severe atrial remodeling, which makes it difficult to get rhythm control. Better understanding about differential gene expressions between maintained SR and recurrent $\mathrm{AF}$ in the patients with markedly dilated atria may help develop strategies to reduce the recurrence rate after the ablation. In this study we found beyond the effect of atrial size, AF recurrence after the maze surgery is associated with more severe atrial remodeling, presented as more

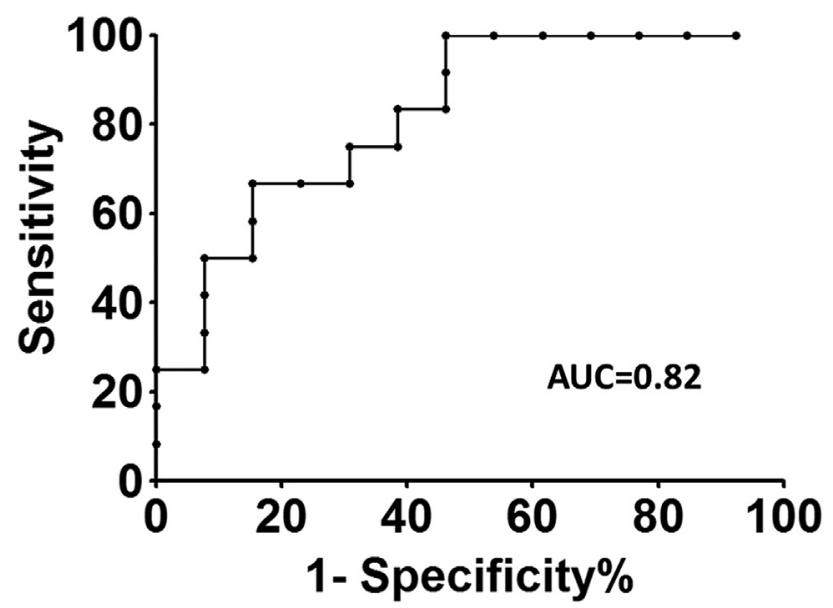

FIGURE 7. Receiver operator curve analysis of $P T K 2$ in recurrent atrial fibrillation after the maze procedure and maintained sinus rhythm after the maze procedure. $A U C$, Area under the curve. intense oxidative stress, upregulations of collagen, TGF$\beta 1$, and differential expression profiles of proinflammatory genes.

\section{Inflammation-Related Gene Remodeling in AF Versus SR}

Our data revealed that several genes were differentially expressed between AF and SR. STATI is a proinflammatory transcription factor downstream to ATIR and PDGFR. In a pig atria tachypacing-induced AF model, both STAT1 and $R A C l$ were upregulated and activated, which means that its pro-AF role seems to involve $R A C l$ signaling. ${ }^{24}$ Echoing findings from the animal study, our data showed that both the expression and activity of STATl were increased in AF compared with SR. Among these genes altered in human AF, as revealed by the array, we demonstrated that in cultured HL-1 atrial myocytes, rapid cellular activation by electric pacing increased the expressions of $R H O A$, $P D G F A$, and TRAF2, whereas the expression of $S H C 1$ was decreased by rapid pacing, suggesting that tachycardia exerts a causal relationship with the expression of these inflammation-related genes. Research has posited RHOA and $P D G F A$ as proinflammatory genes that are associated with $\mathrm{AF}^{25,26}$ The findings from these studies imply that these AF-upregulated genes may therefore participate in the process of $\mathrm{AF}$ begets $\mathrm{AF}$ in atrial substrate remodeling. TRAF2 is a scaffolding protein common to tumor necrosis factor receptors 1 and 2 , and a recent study has demonstrated that TRAF2-mediated cytoprotective effects of tumor necrosis factor in the heart. ${ }^{27}$ This means that the upregulation of TRAF2 in human heart could be protective against AF remodeling. The role of $\mathrm{SHCl}$, on the other hand, is more conflicting. SHCl encodes the protein p66Shc, which has been shown to have prooxidant and proapoptotic activity and to be implicated in the aging process. In contrast, a recent study provided 
evidence that genetic deletion of p66Shc led to increased myocardial infarction in response to short-term ischemia and reperfusion, which means that activation of p66Shc protein seems to represent a protective strategy to prevent ischemic and reperfusion myocardial injury. ${ }^{28}$ The role of $\mathrm{SHCl}$ in AF is still largely unclear.

HSPB1 is small heat shock protein. Overexpression of HSPB1 has been shown to be protective against tachycardia-induced remodeling by attenuation of the RHOA GTPase pathway in in vivo and in vitro models. ${ }^{26,29}$ It has been shown that the induction of heat shock proteins attenuate atrial tachycardia-induced electrical (reduction of expressions of L-type calcium channels) and structural (increased collagen accumulation in the atria) remodeling. Its upregulation in $\mathrm{AF}$ may represent a compensatory mechanism against AF-related remodeling despite that it cannot keep patients in SR in the conditions of severe volume and/or pressure overload due to mitral valve dysfunction.

The characteristics of patients in SR group are different from patients in the AF-SR and AF-AF groups. All the patients in the SR group were diagnosed with nonrheumatic heart disease and more of them had coexisting CAD, diabetes mellitus, and hypertension. Therefore it should be emphasized that the differential gene expressions between SR and AF may not only contribute to cardiac rhythm but also the underlying disease (rheumatic and nonrheumatic). Despite this, we found genes like $S H C l$, RHOA, and PDGFA were upregulated by tachycardia at a cellular tachypacing model of HL-1 cells, which implies a causative relationship from tachycardia to gene changes potentially present.

In the study more patients in the SR group had CAD and therefore were taking aspirin, an anti-inflammatory agent, which may also contribute to the differential proinflammatory gene expressions between SR and AF.

\section{Inflammation-Related Gene Remodeling in Successful Versus Failed Rhythm Control by the Maze Procedure}

Our data firstly showed that several genes were differentially expressed or activated in the atria between patients with maintained SR or recurrent $\mathrm{AF}$ after the maze procedure.

NFAT is a nuclear transcription factor downstream to calcineurin and calcium ion/calmodulin. Activated calcineurin dephosphorylates NFAT and causes its translocation to the nucleus. Numerous studies have reported increased cardiac calcineurin activity and NFAT nuclear translocation in human failing and hypertrophied hearts as well as in arrhythmia animal models. ${ }^{30,31}$ It has been shown in canine atrial myocytes that NFAT is activated by rapid electrical stimulation mimicking the condition of atrial tachycardia, which mediates tachypacing-induced Cav1.2 downregulation and explains the electric mechanisms of $\mathrm{AF}$ begets $\mathrm{AF}$ in respect of decreased L-type calcium current in $\mathrm{AF}^{21}$ The same group proposed in another study that the atrial fibroblasts were also activated in an AF-promoting dog model, in which NFAT in the fibroblasts were activated as well and also played a profibrotic role in $\mathrm{AF}^{32}$ Consistent with the previous studies, we observed higher expression of nuclear NFATc3 and increased oxidative stress in patients with recurrent $\mathrm{AF}$ after the maze procedure, indicating that NFAT activation is likely associated more advanced atrial remodeling as well as oxidative stress, which may make it more difficult for rhythm control to be achieved.

Several proinflammatory mediators were shown to be upregulated in AF-AF compared with AF-SR. For example, both CCLA and ILI5 are cytokines upregulated during the inflammatory response and may be involved in recruitment of neutrophils, and both of which may contribute to inflammatory cell infiltration in the atria and worsen atria remodeling in AF. Our results indicated that both CCLA and $I L 15$ were more highly expressed in the group of failed rhythm control, probably reflecting severe inflammation in AF. At present, their roles in the heart remain to be elucidated.

\section{Previous Studies}

Few research groups have investigated the association between atrial substrate remodeling and the outcome of surgical ablation. Saito and colleagues ${ }^{33}$ examined the $^{3}$ morphologic changes in atrial appendages removed during the maze procedure. They found patients with recurrent $\mathrm{AF}$ have more myocyte hypertrophy, nuclear enlargement, bizarre nuclei, and interstitial fibrosis compared with those patients who still maintained SR. Castoguay and colleagues $^{34}$ found both myocyte hypertrophy and interstitial fibrosis were more often seen in the atria of patients with recurrent AF. Wang and colleagues ${ }^{35}$ found that the expression of matrix metalloproteinase- 2 and collagen I were significantly higher in the atria from patients with recurrent $\mathrm{AF}$ compared with the patients with maintained SR. Our findings revealed that a number of proinflammatory genes differentially expressed between $\mathrm{AF}$ and SR and between AF-AF and AF-SR. Consistent with our previous studies in $\mathrm{AF}$ remodeling, ${ }^{20,36}$ we showed that increased oxidative stress, expression of TGF- $\beta 1$, and activation of NFAT may underlie the more severe changes of substrate remodeling changes in the atria that is refractory to surgical ablation. The data may provide a database and clues to search for potential therapeutic targets in preventing atrial remodeling progression and managing $\mathrm{AF}$.

Nuclear factor- $\kappa \mathrm{B}(\mathrm{NF}-\kappa \mathrm{B})$ is a nuclear transcription factor coupling redox state to changes in gene transcriptional regulation. It has been shown that $\mathrm{NF}-\kappa \mathrm{B}$ was associated 
with $\mathrm{AF}$, and increased oxidative stress reduced the expression of cardiac sodium channel via NF- $\kappa \mathrm{B} .{ }^{37}$ However, we found that $\mathrm{NF}-\kappa \mathrm{B}$ was downregulated in $\mathrm{AF}$ compared with SR (Figure 1, A). Another example is STATl, which is thought to be pro-oxidative and has been shown to contribute to AF in the literature. Our results indicate that although STAT1 was upregulated in AF, its expression was higher in AF-SR compared with AF-AF, which is not consistent with our findings of the more severe remodeling in AF-AF atria. We believe that the interplay between these mediators and $\mathrm{AF}$ and its underlying disease is complex and cannot be simply interpreted according to our observations in this study. More detailed studies are required to explore their relationship with AF pathogenesis.

The inflammatory process is broadly diverse and contains numerous signaling pathways and mediators. Considering age as well as underlying systemic and cardiovascular disease in humans and the complex interaction between them, it is likely that not all the inflammatory processes are equally or concordantly involved in a diseased atria. This may explain why we revealed only a portion of proinflammatory genes were differentially expressed among the genes screened in this study.

\section{Study Limitations}

We applied the cryoablation, therefore AF recurrence could be contributed by incomplete transmural lesion. We minimized the confounding possibility by limited duration to enroll the patients, employing a single operator, and comparing patients with atria dilated to a similar degree. Further studies are needed to verify our hypotheses that more severe atrial remodeling and differential gene changes are associated with recurrent AF in patients with markedly dilated atria. AF is a complex disease, involving a number of etiologic factors and arrhythmia mechanisms. ${ }^{38}$ The generalizability of the results of this study is limited by the relatively small sample size of patients. When considering differences in age, stages of heart function, and drug therapy in human AF, the interpatient variability may be a potentially significant confounding factor. Although our findings cannot represent the whole profile of AF in humans based on the limited patient number, this study includes patients with severe mitral valve dysfunction and longstanding persistent $\mathrm{AF}$, which represent a subgroup of $\mathrm{AF}$, from the view of rhythm control, being among the most severe forms. Our findings are thus valuable to provide potential therapeutic targets when we wish to cure difficult AF. We did not approach the systemic inflammation of the study patients, which may influence on the properties of the atria in addition to AF. Lastly, we did not report the duration of AF because it was not available or clear in some cases. However, in this study all of the cases of AF were more than 1 year's duration.

\section{CONCLUSIONS}

Our study provides new data distinguishing AF and SR and successful and failed (or difficult) restoration of sinus rhythm after maze surgery. These may provide a clue for further data mining and hypothesis generation in $\mathrm{AF}$, which may help improve current modalities in treating AF.

\section{Conflict of Interest Statement}

Authors have nothing to disclose with regard to commercial support.

The authors thank Chih-Chun Chen for providing technical assistance for confocal immunofluorescence in the Microscope Core Laboratory, Chang-Gung Memorial Hospital, Linkou.

\section{References}

1. Woods CE, Olgin J. Atrial fibrillation therapy now and in the future: drugs, biologicals, and ablation. Circ Res. 2014;114:1532-46.

2. Nattel S, Guasch E, Savelieva I, Cosio FG, Valverde I, Halperin JL, et al. Early management of atrial fibrillation to prevent cardiovascular complications. Eur Heart J. 2014;35:1448-56.

3. Nattel S, Maguy A, Le Bouter S, Yeh YH. Arrhythmogenic ion-channel remodeling in the heart: heart failure, myocardial infarction, and atrial fibrillation. Physiol Rev. 2007;87:425-56.

4. Deshmukh A, Barnard J, Sun H, Newton D, Castel L, Pettersson G, et al. Left atrial transcriptional changes associated with atrial fibrillation susceptibility and persistence. Circ Arrhythm Electrophysiol. 2015;8:32-41.

5. Yeh YH, Kuo CT, Lee YS, Lin YM, Nattel S, Tsai FC, et al. Region-specific gene expression profiles in the left atria of patients with valvular atrial fibrillation. Heart Rhythm. 2013;10:383-91

6. Wakili R, Voigt N, Kaab S, Dobrev D, Nattel S. Recent advances in the molecular pathophysiology of atrial fibrillation. J Clin Invest. 2011;121:2955-68.

7. Gillinov AM. Advances in surgical treatment of atrial fibrillation. Stroke. 2007; 38(2 Suppl):618-23.

8. Cox JL, Schuessler RB, D'Agostino HJ Jr, Stone CM, Chang BC, Cain ME, et al. The surgical treatment of atrial fibrillation. III. Development of a definitive surgical procedure. J Thorac Cardiovasc Surg. 1991;101:569-83.

9. Beukema WP, Sie HT, Misier AR, Delnoy PP, Wellens HJ, Elvan A. Intermediate to long-term results of radiofrequency modified Maze procedure as an adjunct to open-heart surgery. Ann Thorac Surg. 2008;86:1409-14.

10. Ad N, Henry L, Massimiano P, Pritchard G, Holmes SD. The state of surgical ablation for atrial fibrillation in patients with mitral valve disease. Curr Opin Cardiol. 2013;28:170-80.

11. Ad N, Holmes SD, Massimiano PS, Pritchard G, Stone LE, Henry L. The effect of the Cox-maze procedure for atrial fibrillation concomitant to mitral and tricuspid valve surgery. J Thorac Cardiovasc Surg. 2013;146:1426-34; discussion 34-5.

12. Gillinov AM, Gelijns AC, Parides MK, DeRose JJ Jr, Moskowitz AJ, Voisine P et al. Surgical ablation of atrial fibrillation during mitral-valve surgery. $N$ Engl J Med. 2015;372:1399-409.

13. Ad N, Holmes SD, Pritchard G, Shuman DJ. Association of operative risk with the outcome of concomitant Cox Maze procedure: a comparison of results across risk groups. J Thorac Cardiovasc Surg. 2014;148:3027-33.

14. Stulak JM, Suri RM, Burkhart HM, Daly RC, Dearani JA, Greason KL, et al Surgical ablation for atrial fibrillation for two decades: are the results of new techniques equivalent to the Cox maze III procedure? J Thorac Cardiovasc Surg. 2014; 147:1478-86.

15. Ad N, Henry L, Hunt S, Holmes SD. Should surgical ablation for atrial fibrillation be performed in patients with a significantly enlarged left atrium? J Thorac Cardiovasc Surg. 2014;147:236-41.

16. Wolke C, Bukowska A, Goette A, Lendeckel U. Redox control of cardiac remodeling in atrial fibrillation. Biochim Biophys Acta. 2014;1850:1555-65.

17. Hu YF, Chen YJ, Lin YJ, Chen SA. Inflammation and the pathogenesis of atrial fibrillation. Nat Rev Cardiol. 2015;12:230-43.

18. Friedrichs K, Klinke A, Baldus S. Inflammatory pathways underlying atrial fibrillation. Trends Mol Med. 2011;17:556-63. 
19. Yeh YH, Kuo CT, Chang GJ, Chen YH, Lai YJ, Cheng ML, et al. Rosuvastatin suppresses atrial tachycardia-induced cellular remodeling via Akt/Nrf2/heme oxygenase-1 pathway. J Mol Cell Cardiol. 2015;82:84-92.

20. Yeh YH, Kuo CT, Chang GJ, Qi XY, Nattel S, Chen WJ. Nicotinamide adenine dinucleotide phosphate oxidase 4 mediates the differential responsiveness of atrial versus ventricular fibroblasts to transforming growth factor-beta. Circ Arrhythm Electrophysiol. 2013;6:790-8.

21. Qi XY, Yeh YH, Xiao L, Burstein B, Maguy A, Chartier D, et al. Cellular signaling underlying atrial tachycardia remodeling of L-type calcium current. Circ Res. 2008;103:845-54.

22. Beukema WP, Sie HT, Misier AR, Delnoy PP, Wellens HJ, Elvan A. Predictive factors of sustained sinus rhythm and recurrent atrial fibrillation after a radiofrequency modified Maze procedure. Eur J Cardiothorac Surg. 2008;34: $771-5$.

23. Ad N, Holmes SD. Prediction of sinus rhythm in patients undergoing concomitant Cox maze procedure through a median sternotomy. J Thorac Cardiovasc Surg. 2014;148:881-6; discussion 6-7.

24. Tsai CT, Lin JL, Lai LP, Lin CS, Huang SK. Membrane translocation of small GTPase Rac1 and activation of STAT1 and STAT3 in pacing-induced sustained atrial fibrillation. Heart Rhythm. 2008;5:1285-93.

25. Liao CH, Akazawa H, Tamagawa M, Ito K, Yasuda N, Kudo Y, et al. Cardiac mast cells cause atrial fibrillation through PDGF-A-mediated fibrosis in pressure-overloaded mouse hearts. J Clin Invest. 2009;120:242-53.

26. Ke L, Meijering RA, Hoogstra-Berends F, Mackovicova K, Vos MJ, Van Gelder IC, et al. HSPB1, HSPB6, HSPB7 and HSPB8 protect against RhoA GTPase-induced remodeling in tachypaced atrial myocytes. PLoS One. 2011; 6:e20395.

27. Burchfield JS, Dong JW, Sakata Y, Gao F, Tzeng HP, Topkara VK, et al. The cytoprotective effects of tumor necrosis factor are conveyed through tumor necrosis factor receptor-associated factor 2 in the heart. Circ Heart Fail. 2010; 3:157-64.

28. Akhmedov A, Montecucco F, Braunersreuther V, Camici GG, Jakob P, Reiner MF, et al. Genetic deletion of the adaptor protein p66Shc increases susceptibility to short-term ischaemic myocardial injury via intracellular salvage pathways. Eur Heart J. 2015;36:516-526a.
29. Brundel BJ, Shiroshita-Takeshita A, Qi X, Yeh YH, Chartier D, van Gelder IC et al. Induction of heat shock response protects the heart against atrial fibrillation. Circ Res. 2006;99:1394-402.

30. Molkentin JD. Calcineurin-NFAT signaling regulates the cardiac hypertrophic response in coordination with the MAPKs. Cardiovasc Res. 2004;63:467-75.

31. Qi X, Yeh YH, Chartier D, Xiao L, Tsuji Y, Brundel BJ, et al. The calcium/ calmodulin/kinase system and arrhythmogenic afterdepolarizations in bradycardia-related acquired long-QT syndrome. Circ Arrhythm Electrophysiol. 2009;2:295-304.

32. Harada M, Luo X, Qi XY, Tadevosyan A, Maguy A, Ordog B, et al. Transient receptor potential canonical-3 channel-dependent fibroblast regulation in atrial fibrillation. Circulation. 2012;126:2051-64

33. Saito T, Tamura K, Uchida D, Togashi M, Nitta T, Sugisaki Y. Histopathological features of the resected left atrial appendage as predictors of recurrence after surgery for atrial fibrillation in valvular heart disease. Circ J. 2007;71:70-8

34. Castonguay MC, Wang Y, Gerhart JL, Miller DV, Stulak JM, Edwards WD, et al. Surgical pathology of atrial appendages removed during the cox-maze procedure: a review of 86 cases (2004 to 2005) with implications for prognosis. Am J Surg Pathol. 2013;37:890-7.

35. Wang X, Li Y, Liu L, Hu SS, Song YH, Wang W. The role of matrix metalloproteinase- 2 in the treatment of atrial fibrillation recurrence after a radiofrequency modified maze procedure. Cardiology. 2013;126:62-8.

36. Yeh YH, Kuo CT, Chan TH, Chang GJ, Qi XY, Tsai F, et al. Transforming growth factor-beta and oxidative stress mediate tachycardia-induced cellular remodelling in cultured atrial-derived myocytes. Cardiovasc Res. 2011;91: 62-70.

37. Gao G, Dudley SC Jr. Redox regulation, NF-kappaB, and atrial fibrillation. Antioxid Redox Signal. 2009;11:2265-77.

38. Yeh YH, Wakili R, Qi XY, Chartier D, Boknik P, Kaab S, et al. Calcium-handling abnormalities underlying atrial arrhythmogenesis and contractile dysfunction in dogs with congestive heart failure. Circ Arrhythm Electrophysiol. 2008;1:93-102.

Key Words: atrial fibrillation, maze, NFAT, inflammation 


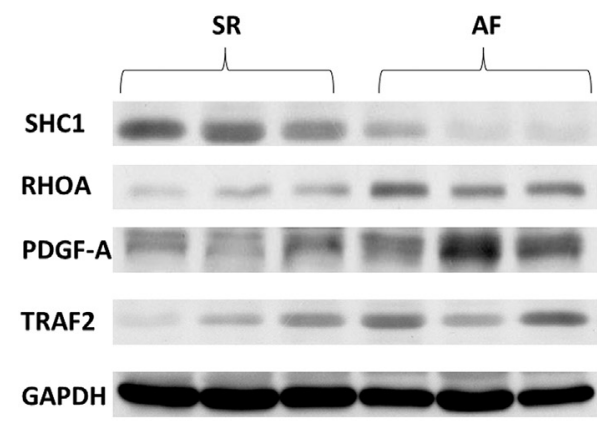

A

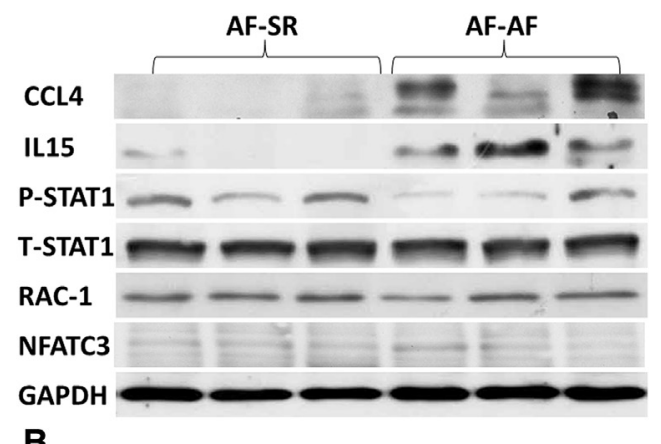

B

FIGURE E1. A, Representative examples of Western blot of $S H C 1, R H O A, P D G F A$, and TRAF2 between sinus rhythm $(S R)$ and atrial fibrillation $(A F)$ and B, Representative examples of Wetern blot of CCL4, IL15, phospho-, and total STAT1, RAC1, and nuclear factor of activated T-cells cytoplasmic 3 (NFATC3) between recurrent atrial fibrillation after the maze procedure $(A F-A F)$ and maintained sinus rhythm after maze procedure $(A F-S R)$.

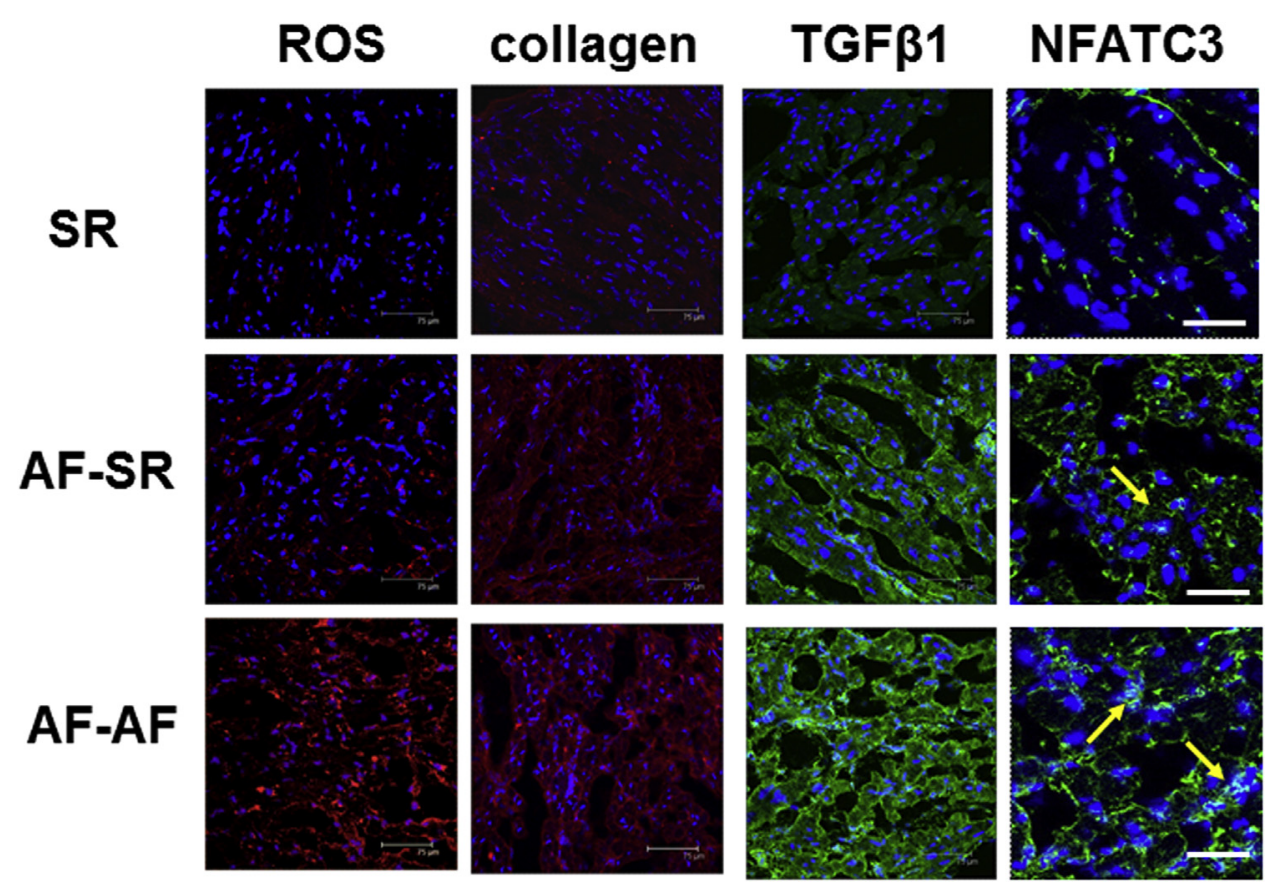

FIGURE E2. Representative examples of confocal immunohistochemistry of reactive oxidative stress (ROS), transforming growth factor beta 1 (TGF- $\beta 1$ ), collagen, and nuclear factor of activated T-cells cytoplasmic 3 (NFATc3) between sinus rhythm $(S R)$, maintained sinus rhythm after maze procedure ( $A F-S R)$ and recurrent atrial fibrillation after maze procedure $(A F-A F)$. 


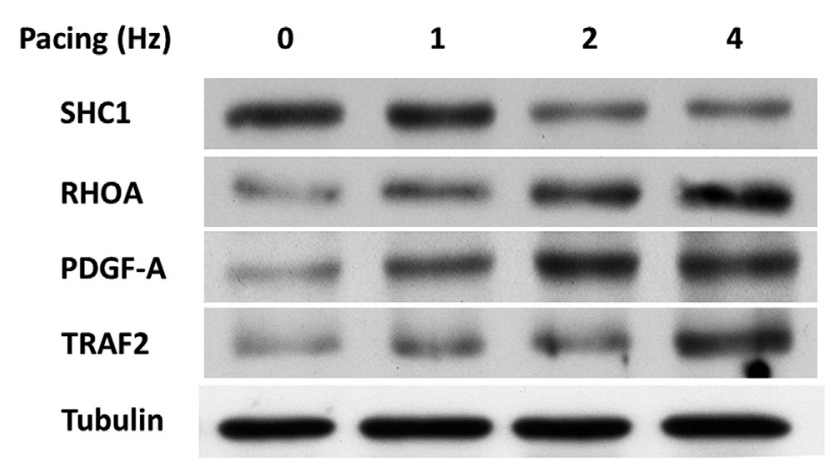

FIGURE E3. Representative examples of Western blot of $S H C 1$, RHOA, $P D G F-A$, and TRAF2 in HL-1 atrial myocyte after pacing.

TABLE E1. List of primers applied by quantitative polymerase chain reaction in left atrial appendage

\begin{tabular}{|c|c|c|}
\hline Gene & Genebank ID & Primer sequence \\
\hline CCL4 & NM_002984.2 & Forward: 5'-GCTTCCTCGCAACTTTGTGG-3' Reverse: 5'-TCACTGGGATCAGCACAGAC-3' \\
\hline $\mathrm{CFB}$ & NM_001710 & Forward: 5'-ACAACAGAAGCGGAAGAT-3' Reverse: 5'-ACCATAACTTGCCACCTT-3' \\
\hline CFD & NM_001928 & Forward: 5'-GCGACTCCATCTCTACAA-3' Reverse: 5'-CTGCGTTCAAGTCATCCT-3' \\
\hline CXCL6 & NM_002993.3 & Forward: 5'-CCTCTCTTGACCACTATGAG-3' Reverse: 5'-GTTTTGGGGTTTACTCTCAG-3' \\
\hline GNAS & NM_080425 & Forward: 5'-CAACTTACTCCGCAACTT-3' Reverse: 5'-ACCTTCTTGACCTTGAGA-3' \\
\hline HRAS & NM_005343.2 & Forward: 5'-ACCATTTTGTGGACGAATAC-3' Reverse: 5'-AAGACTTGGTGTTGTTGATG-3' \\
\hline HSPB1 & NM_001540 & Forward: 5'-AAACGGGTCATTGCCATTA-3' Reverse: 5'-CGCTTTTATGCTCCTCCA-3' \\
\hline IL10 & NM_000572.2 & Forward: 5'-TCCAGTGTCTCGGAGGGATT-3' Reverse: 5'-TGGCCACAGCTTTCAAGAATG-3' \\
\hline IL15 & NM_172174 & Forward: 5'-CTACTGGCTCTCATTGACT-3' Reverse: 5'-GAGGATTCACATATAGTTCTTCTT-3' \\
\hline IL18 & NM_001562.2 & Forward: 5'-CCTTTAAGGAAATGAATCCTCC-3' Reverse: 5'-CATCTTATTATCATGTCCTGGG-3' \\
\hline IL1B & NM_000576 & Forward: 5'-TACATCAGCACCTCTCAAG-3' Reverse: 5'-ATTCAGCACAGGACTCTC-3' \\
\hline MEF2A & NM_005587 & Forward: 5'-GAACTACTTACAGGCACATT-3' Reverse: 5'-ACGAAGGAATCACTTAACAC-3' \\
\hline PDGFA & NM_002607.5 & Forward: 5'-CGCAGGAAGCAGGAATGTCT-3' Reverse: 5'-ACAGCGAGGAGGTGTGGTTT-3' \\
\hline PPP1R12B & NM_032103.1 & Forward: 5'-GGGCCAGAAGTAGCTTGAC-3' Reverse: 5'-TGTTCCGGGTTTCAGTAGAGT-3' \\
\hline RHOA & NM_001664.2 & Forward: 5'-GCTGGGCTAAGTAAATAGGAATTGG-3' Reverse: 5'-GTCCAGGTGAGACAGGTTATGC-3' \\
\hline SHC1 & NM_183001.4 & Forward: 5'-GCGAGACTGAGAGGCAGAA-3' Reverse: 5'-CCGGGCCTGTAGAAGGTACTC-3' \\
\hline STAT1 & NM_007315.2 & Forward: 5'-TGCTGAGGTTTAGCTGTCAGTTC-3' Reverse: 5'-GTCTCTTGGCTAGTGCAGTAAA-3' \\
\hline
\end{tabular}


TABLE E2. List of genes detected by nCounter Human Inflammation Array (NanoString Technologies, Seattle, Wash) in left atrial appendage

\begin{tabular}{|c|c|c|}
\hline Gene symbol & GeneBank Accession & Gene name \\
\hline \multicolumn{3}{|l|}{ Cytokine } \\
\hline CCL11 & NM_002986.2 & chemokine (C-C motif) ligand 11 \\
\hline CCL13 & NM_005408.2 & chemokine (C-C motif) ligand 13 \\
\hline CCL19 & NM_006274.2 & chemokine (C-C motif) ligand 19 \\
\hline CCL2 & NM_002982.3 & chemokine (C-C motif) ligand 2 \\
\hline CCL21 & NM_002989.2 & chemokine (C-C motif) ligand 21 \\
\hline CCL24 & NM_002991.2 & chemokine (C-C motif) ligand 24 \\
\hline CCL3 & NM_002983.2 & chemokine (C-C motif) ligand 3 \\
\hline CCL4 & NM_002984.2 & chemokine (C-C motif) ligand 4 \\
\hline CCL5 & NM_002985.2 & chemokine (C-C motif) ligand 5 \\
\hline CCL8 & NM_005623.2 & chemokine (C-C motif) ligand 8 \\
\hline CXCL1 & NM_001511.1 & chemokine (C-X-C motif) ligand 1 \\
\hline CXCL10 & NM_001565.1 & chemokine (C-X-C motif) ligand 10 \\
\hline CXCL2 & NM_002089.1 & chemokine (C-X-C motif) ligand 2 \\
\hline CXCL3 & NM_002090.2 & chemokine (C-X-C motif) ligand 3 \\
\hline CXCL6 & NM_002993.3 & chemokine (C-X-C motif) ligand 6 \\
\hline CXCL9 & NM_002416.1 & chemokine (C-X-C motif) ligand 9 \\
\hline IL1B & NM_000576.2 & interleukin 1 , beta \\
\hline IL10 & NM_000572.2 & interleukin 10 \\
\hline IL15 & NM_172174.1 & interleukin 15 \\
\hline IL18 & NM_001562.2 & interleukin 18 \\
\hline IL6 & NM_000600.1 & interleukin 6 \\
\hline IL7 & NM_000880.2 & interleukin 7 \\
\hline IL8 & NM_000584.2 & interleukin 8 \\
\hline LTB & NM_002341.1 & lymphotoxin beta \\
\hline PDGFA & NM_002607.5 & platelet-derived growth factor alpha polypeptide \\
\hline TGFB1 & NM_000660.3 & transforming growth factor, beta 1 \\
\hline TGFB2 & NM_003238.2 & transforming growth factor, beta 2 \\
\hline TGFB3 & NM_003239.2 & transforming growth factor, beta 3 \\
\hline TNF & NM_000594.2 & tumor necrosis factor \\
\hline \multicolumn{3}{|l|}{ Receptor } \\
\hline CD4 & NM_000616.3 & CD4 molecule \\
\hline $\mathrm{CD} 40$ & NM_001250.4 & CD40 molecule, TNF receptor superfamily member 5 \\
\hline CD55 & NM_000574.3 & CD55 molecule, decay accelerating factor for complement \\
\hline $\mathrm{CDC} 42$ & NM_001039802.1 & cell division cycle 42 \\
\hline CCR1 & NM_001295.2 & chemokine (C-C motif) receptor 1 \\
\hline CXCR4 & NM_003467.2 & chemokine (C-X-C motif) receptor 4 \\
\hline C3AR1 & NM_004054.2 & complement component $3 \mathrm{a}$ receptor 1 \\
\hline DAXX & NM_001350.3 & death-domain associated protein \\
\hline GRB2 & NM_203506.2 & growth factor receptor-bound protein 2 \\
\hline HLA-DRB1 & NM_002124.1 & major histocompatibility complex, class II, DR beta 1 \\
\hline IL1RAP & NM_002182.2 & interleukin 1 receptor accessory protein \\
\hline IL1RN & NM_173842.1 & interleukin 1 receptor antagonist \\
\hline IL1R1 & NM_000877.2 & interleukin 1 receptor, type I \\
\hline IL10RB & NM_000628.3 & interleukin 10 receptor, beta \\
\hline IL18RAP & NM_003853.2 & interleukin 18 receptor accessory protein \\
\hline IL6R & NM_000565.2 & interleukin 6 receptor \\
\hline IL8RA & NM_000634.2 & interleukin 8 receptor, alpha \\
\hline IL8RB & NM_001557.2 & interleukin 8 receptor, beta \\
\hline LY96 & NM_015364.2 & lymphocyte antigen 96 \\
\hline HLA-DRA & NM_019111.3 & major histocompatibility complex, class II, DR alpha \\
\hline SHC1 & NM_183001.4 & SHC (Src homology 2 domain containing) transforming protein 1 \\
\hline TLR1 & NM_003263.3 & toll-like receptor 1 \\
\hline TLR2 & NM_003264.3 & toll-like receptor 2 \\
\hline TLR3 & NM_003265.2 & toll-like receptor 3 \\
\hline
\end{tabular}


TABLE E2. Continued

\begin{tabular}{|c|c|c|}
\hline Gene symbol & GeneBank Accession & Gene name \\
\hline TLR4 & NM_138554.2 & toll-like receptor 4 \\
\hline TLR5 & NM_003268.3 & toll-like receptor 5 \\
\hline TLR6 & NM_006068.2 & toll-like receptor 6 \\
\hline TLR7 & NM_016562.3 & toll-like receptor 7 \\
\hline TGFBR1 & NM_004612.2 & transforming growth factor beta receptor 1 \\
\hline \multicolumn{3}{|c|}{ Signal transduction } \\
\hline CREB1 & NM_134442.2 & cAMP responsive element binding protein 1 \\
\hline CLTC & NM_004859.2 & clathrin, heavy chain $(\mathrm{Hc})$ \\
\hline CFL1 & NM_005507.2 & cofilin 1 \\
\hline CSF1 & NM_000757.4 & colony stimulating factor 1 \\
\hline C1QA & NM_015991.2 & complement component 1 , q subcomponent, A chain \\
\hline C1QB & NM_000491.3 & complement component 1 , q subcomponent, B chain \\
\hline $\mathrm{C} 1 \mathrm{R}$ & NM_001733.4 & complement component $1, \mathrm{r}$ subcomponent \\
\hline $\mathrm{C} 1 \mathrm{~S}$ & NM_001734.2 & complement component $1, \mathrm{~s}$ subcomponent \\
\hline $\mathrm{C} 2$ & NM_000063.3 & complement component 2 \\
\hline $\mathrm{C} 3$ & NM_000064.2 & complement component 3 \\
\hline $\mathrm{C} 4 \mathrm{~A}$ & NM_007293.2 & complement component $4 \mathrm{~A}$ \\
\hline C5 & NM_001735.2 & complement component 5 \\
\hline C6 & NM_000065.2 & complement component 6 \\
\hline $\mathrm{C} 7$ & NM_000587.2 & complement component 7 \\
\hline CFB & NM_001710.5 & complement factor B \\
\hline FOS & NM_005252.2 & FBJ murine osteosarcoma viral oncogene homolog \\
\hline GUSB & NM_000181.1 & glucuronidase, beta \\
\hline GAPDH & NM_002046.3 & glyceraldehyde-3-phosphate dehydrogenase \\
\hline GNAS & NM_080425.1 & GNAS complex locus \\
\hline GNB1 & NM_002074.2 & guanine nucleotide binding protein ( $\mathrm{G}$ protein), beta polypeptide 1 \\
\hline GNAQ & NM_002072.2 & guanine nucleotide binding protein ( $\mathrm{G}$ protein), q polypeptide \\
\hline HSPB1 & NM_001540.3 & heat shock $27 \mathrm{kDa}$ protein 1 \\
\hline HSPB2 & NM_001541.3 & heat shock $27 \mathrm{kDa}$ protein 2 \\
\hline HPRT1 & NM_000194.1 & hypoxanthine phosphoribosyltransferase 1 \\
\hline CFD & NM_001928.2 & complement factor B \\
\hline FXYD2 & NM_021603.3 & FBJ murine osteosarcoma viral oncogene homolog \\
\hline MAFK & NM_002360.3 & v-maf avian musculoaponeurotic fibrosarcoma oncogene homolog $\mathrm{K}$ \\
\hline MYC & NM_002467.3 & MYC associated factor $\mathrm{X}$ \\
\hline ITGB2 & NM_000211.2 & integrin, beta 2 \\
\hline KEAP1 & NM_012289.3 & kelch-like ECH-associated protein 1 \\
\hline LIMK1 & NM_002314.2 & LIM domain kinase 1 \\
\hline MASP1 & NM_139125.2 & mannan-binding lectin serine peptidase 1 \\
\hline MKNK1 & NM_003684.3 & MAP kinase interacting serine/threonine kinase 1 \\
\hline MAPK1 & NM_002745.4 & mitogen-activated protein kinase 1 \\
\hline MAPK14 & NM_001315.1 & mitogen-activated protein kinase 14 \\
\hline MAPK3 & NM_001040056.1 & mitogen-activated protein kinase 3 \\
\hline MAPK8 & NM_002750.2 & mitogen-activated protein kinase 8 \\
\hline MAP2K1 & NM_002755.2 & mitogen-activated protein kinase kinase 1 \\
\hline MAP2K4 & NM_003010.2 & mitogen-activated protein kinase kinase 4 \\
\hline MAP2K6 & NM_002758.3 & mitogen-activated protein kinase kinase 6 \\
\hline MAP3K1 & NM_005921.1 & mitogen-activated protein kinase kinase kinase 1, E3 ubiquitin protein ligase \\
\hline MAP3K5 & NM_005923.3 & mitogen-activated protein kinase kinase kinase 5 \\
\hline MAP3K7 & NM_145333.1 & mitogen-activated protein kinase kinase kinase 7 \\
\hline MAPKAPK2 & NM_004759.3 & mitogen-activated protein kinase-activated protein kinase 2 \\
\hline MAPKAPK5 & NM_003668.2 & mitogen-activated protein kinase-activated protein kinase 5 \\
\hline MAX & NM_145113.1 & MYC-associated factor X \\
\hline MYD88 & NM_002468.3 & myeloid differentiation primary response gene (88) \\
\hline MYL2 & NM_000432.3 & myosin, light chain 2 , regulatory, cardiac, slow \\
\hline NOS2 & NM_000625.4 & nitric oxide synthase 2 , inducible \\
\hline
\end{tabular}


TABLE E2. Continued

\begin{tabular}{|c|c|c|}
\hline Gene symbol & GeneBank Accession & Gene name \\
\hline $\mathrm{NR} 3 \mathrm{C} 1$ & NM_001018074.1 & nuclear receptor subfamily 3 , group $\mathrm{C}$, member 1 \\
\hline PGK1 & NM_000291.2 & phosphoglycerate kinase 1 \\
\hline PLA2G4A & NM_024420.2 & phospholipase A2, group IVA \\
\hline PLCB1 & NM_182734.1 & phospholipase $\mathrm{C}$, beta 1 \\
\hline PRKCA & NM_002737.2 & protein kinase $\mathrm{C}$, alpha \\
\hline PRKCB & NM_212535.1 & protein kinase $\mathrm{C}$, beta \\
\hline PPP1R12B & NM_032103.1 & protein phosphatase 1 , regulatory subunit $12 \mathrm{~B}$ \\
\hline PTK2 & NM_005607.3 & PTK2 protein tyrosine kinase 2 \\
\hline RAPGEF2 & NM_014247.2 & Rap guanine nucleotide exchange factor (GEF) 2 \\
\hline RHOA & NM_001664.2 & ras homolog family member $\mathrm{A}$ \\
\hline RAC1 & NM_198829.1 & ras-related $\mathrm{C} 3$ botulinum toxin substrate 1 \\
\hline RIPK1 & NM_003804.3 & receptor (TNFRSF)-interacting serine-threonine kinase 1 \\
\hline RIPK2 & NM_003821.5 & receptor-interacting serine-threonine kinase 2 \\
\hline ROCK2 & NM_004850.3 & Rho-associated, coiled-coil containing protein kinase 2 \\
\hline RPS6KA5 & NM_004755.2 & ribosomal protein S6 kinase, 90kDa, polypeptide 5 \\
\hline TRAF2 & NM_021138.3 & TNF receptor-associated factor 2 \\
\hline TRADD & NM_003789.2 & TNFRSF1A-associated via death domain \\
\hline TOLLIP & NM_019009.2 & toll interacting protein \\
\hline HRAS & NM_005343.2 & v-Ha-ras Harvey rat sarcoma viral oncogene homolog \\
\hline RAF1 & NM_002880.2 & v-raf-1 murine leukemia viral oncogene homolog 1 \\
\hline ATF2 & NM_001880.2 & activating transcription factor 2 \\
\hline BCL6 & NM_001706.2 & B-cell CLL/lymphoma 6 \\
\hline CEBPB & NM_005194.2 & CCAAT/enhancer binding protein (C/EBP), beta \\
\hline DDIT3 & NM_004083.4 & DNA-damage-inducible transcript 3 \\
\hline ELK1 & NM_005229.3 & ELK1, member of ETS oncogene family \\
\hline HMGN1 & NM_004965.6 & high mobility group nucleosome binding domain 1 \\
\hline \multicolumn{3}{|c|}{ Transcription factor } \\
\hline HDAC4 & NM_006037.3 & histone deacetylase 4 \\
\hline JUN & NM_002228.3 & jun proto-oncogene \\
\hline MEF2A & NM_005587.2 & myocyte enhancer factor $2 \mathrm{~A}$ \\
\hline MEF2C & NM_002397.3 & myocyte enhancer factor $2 \mathrm{C}$ \\
\hline MEF2D & NM_005920.2 & myocyte enhancer factor $2 \mathrm{D}$ \\
\hline NFE2L2 & NM_006164.3 & nuclear factor (erythroid-derived 2)-like 2 \\
\hline NFATC3 & NM_004555.2 & nuclear factor of activated T-cells, cytoplasmic, calcineurin-dependent 3 \\
\hline NFKB1 & NM_003998.2 & nuclear factor of kappa light polypeptide gene enhancer in B-cells 1 \\
\hline STAT1 & NM_007315.2 & signal transducer and activator of transcription $1,91 \mathrm{kDa}$ \\
\hline MAFF & NM_012323.2 & v-maf musculoaponeurotic fibrosarcoma oncogene homolog F \\
\hline MAFG & NM_002359.2 & v-maf musculoaponeurotic fibrosarcoma oncogene homolog $G$ \\
\hline
\end{tabular}

